Supporting Information

\title{
Mesoscopic Architectures Made of Electrically Charged Binary Colloidal Nanosheets in Aqueous System
}

Teruyuki Nakato, ${ }^{* 1,2}$ Atsushi Takahashi, ${ }^{3}$ Shinya Terada, ${ }^{1}$ Shogo Yamaguchi, ${ }^{1}$ Emiko Mouri, ${ }^{1,2}$ Morio Shintate, ${ }^{4}$ Shinya Yamamoto, ${ }^{4}$ Yusuke Yamauchi, ${ }^{5}$ and Nobuyoshi Miyamoto*4

${ }^{1}$ Department of Applied Chemistry, Kyushu Institute of Technology, 1-1 Sensui-cho, Tobata, Kitakyushu, Fukuoka 804-8550, Japan

2 Strategic Research Unit for Innovative Multiscale Materials, Kyushu Institute of Technology, 1-1 Sensui-cho, Tobata, Kitakyushu, Fukuoka 804-8550, Japan

${ }^{3}$ Graduate School of Bio-Applications and Systems Engineering, Tokyo University of Agriculture and Technology, 2-24-16 Naka-cho, Koganei, Tokyo 184-8588, Japan

4 Department of Life, Environment, and Applied Chemistry, Faculty of Engineering, Fukuoka Institute of Technology, 3-30-1 Wajiro-higashi, Higashi-ku, Fukuoka 811-0295, Japan

5 School of Chemical Engineering and Australian Institute for Bioengineering and Nanotechnology (AIBN), The University of Queensland, Brisbane, QLD 4072 Australia 

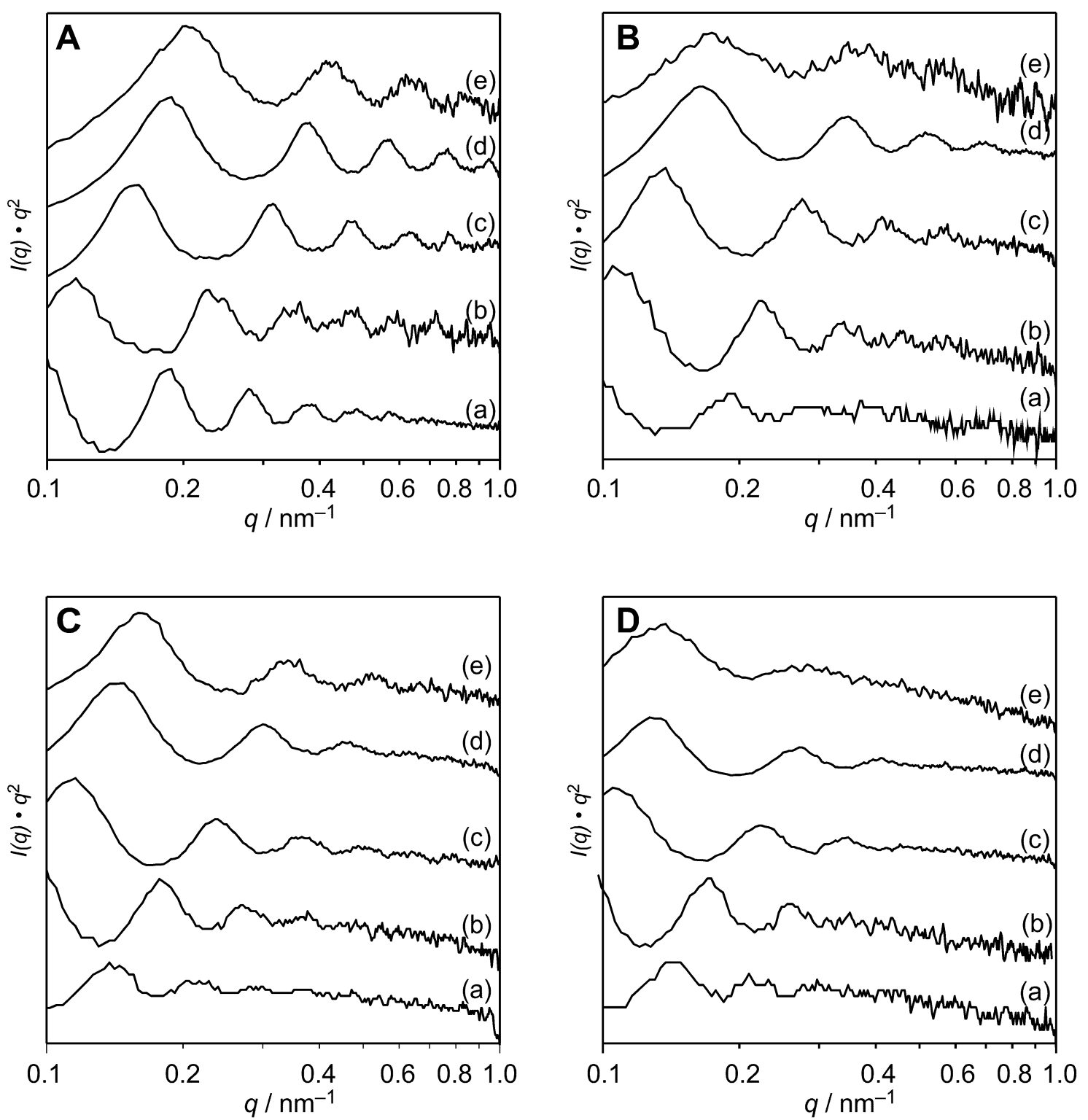

Figure S1. SAXS patterns of NB-clay binary nanosheet colloids at NB concentration of $20 \mathrm{~g} \mathrm{~L}^{-1}$. Both axes are in log-scale. (A) NB-Hect binary colloids with the clay concentration of (a) 5, (b) 10, (c) 20, (d) 30, and (e) $40 \mathrm{~g} \mathrm{~L}^{-1}$. (B) NB-Sapo binary colloids with the clay concentration of (a) 5, (b) 10, (c) 20, (d) 30 , and (e) $40 \mathrm{~g} \mathrm{~L}^{-1}$. NB-FH binary colloids with the clay concentration of (a) 5, (b) 10, (c) 20, (d) 30, and (e) $40 \mathrm{~g} \mathrm{~L}^{-1}$. (D) NB-TSM binary colloids with the clay concentration of (a) 5, (b) 10, (c) 20, (d) 30, and (e) $40 \mathrm{~g} \mathrm{~L}^{-1}$. 

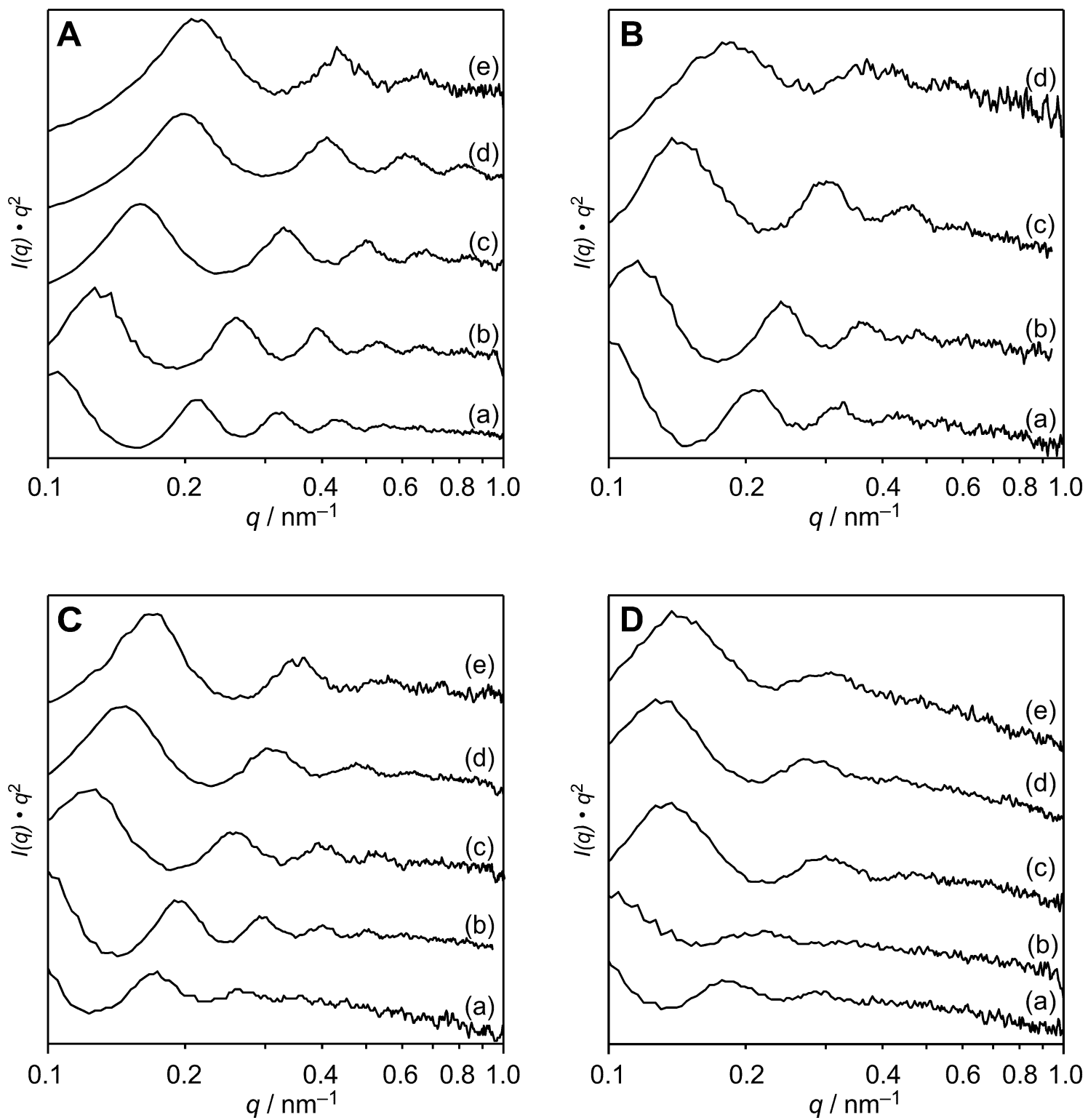

Figure S2. SAXS patterns of NB-clay binary nanosheet colloids at NB concentration of $30 \mathrm{~g} \mathrm{~L}^{-1}$. Both axes are in log-scale. (A) NB-Hect binary colloids with the clay concentration of (a) 5, (b) 10, (c) 20, (d) 30, and (e) $40 \mathrm{~g} \mathrm{~L}^{-1}$. (B) NB-Sapo binary colloids with the clay concentration of (a) 5 , (b) 10 , (c) 20 , and (d) $40 \mathrm{~g} \mathrm{~L}^{-1}$. (C) $\mathrm{NB}-$ FH binary colloids with the clay concentration of (a) 5, (b) 10, (c) 20, (d) 30, and (e) 40 $\mathrm{g} \mathrm{L}^{-1}$. (D) NB-TSM binary colloids with the clay concentration of (a) 5, (b) 10, (c) 20, (d) 30 , and (e) $40 \mathrm{~g} \mathrm{~L}^{-1}$. 
Table S1. Basal spacing of the NB-clay binary colloids.

\begin{tabular}{|c|c|c|c|c|}
\hline Sample & $\mathrm{NB} / \mathrm{g} \mathrm{L}^{-1}$ & Clay / $\mathrm{g} \mathrm{L}^{-1}$ & $\begin{array}{l}\text { Basal spacing } \\
/ \mathrm{nm}\end{array}$ & $\begin{array}{l}\text { Volume occupancy of the NB } \\
\text { lamellar phase in the colloid }\end{array}$ \\
\hline \multirow[t]{19}{*}{ NB-Hect } & \multirow[t]{5}{*}{10} & 5 & 91 & 0.32 \\
\hline & & 10 & 47 & 0.17 \\
\hline & & 20 & 40 & 0.14 \\
\hline & & 30 & 33 & 0.12 \\
\hline & & 40 & 29 & 0.10 \\
\hline & \multirow[t]{5}{*}{20} & 5 & 68 & 0.48 \\
\hline & & 10 & 55 & 0.28 \\
\hline & & 20 & 40 & 0.27 \\
\hline & & 30 & 33 & 0.28 \\
\hline & & 40 & 30 & 0.25 \\
\hline & \multirow[t]{4}{*}{30} & 5 & 60 & 0.64 \\
\hline & & 10 & 53 & 0.51 \\
\hline & & 20 & 44 & 0.33 \\
\hline & & 40 & 34 & 0.31 \\
\hline & \multirow[t]{5}{*}{40} & 5 & 56 & 0.79 \\
\hline & & 10 & 47 & 0.66 \\
\hline & & 20 & 36 & 0.51 \\
\hline & & 30 & 31 & 0.44 \\
\hline & & 40 & 28 & 0.40 \\
\hline \multirow[t]{18}{*}{ NB-Sapo } & \multirow[t]{4}{*}{10} & 5 & 82 & 0.29 \\
\hline & & 10 & 62 & 0.22 \\
\hline & & 20 & 46 & 0.16 \\
\hline & & 30 & 45 & 0.16 \\
\hline & \multirow[t]{5}{*}{20} & 5 & 67 & 0.47 \\
\hline & & 10 & 57 & 0.32 \\
\hline & & 20 & 45 & 0.31 \\
\hline & & 30 & 37 & 0.32 \\
\hline & & 40 & 35 & 0.28 \\
\hline & \multirow[t]{4}{*}{30} & 5 & 60 & 0.64 \\
\hline & & 10 & 53 & 0.61 \\
\hline & & 20 & 44 & 0.47 \\
\hline & & 40 & 34 & 0.36 \\
\hline & \multirow[t]{5}{*}{40} & 5 & 62 & 0.88 \\
\hline & & 10 & 49 & 0.69 \\
\hline & & 20 & 40 & 0.49 \\
\hline & & 30 & 35 & 0.49 \\
\hline & & 40 & 35 & 0.49 \\
\hline \multirow[t]{11}{*}{ NB-FH } & \multirow[t]{4}{*}{10} & 5 & 105 & 0.37 \\
\hline & & 10 & 79 & 0.21 \\
\hline & & 20 & 60 & 0.21 \\
\hline & & 40 & 40 & 0.14 \\
\hline & \multirow[t]{5}{*}{20} & 5 & 89 & 0.63 \\
\hline & & 10 & 71 & 0.37 \\
\hline & & 20 & 53 & 0.35 \\
\hline & & 30 & 40 & 0.37 \\
\hline & & 40 & 37 & 0.36 \\
\hline & \multirow[t]{2}{*}{30} & 5 & 72 & 0.76 \\
\hline & & 10 & 64 & 0.68 \\
\hline
\end{tabular}


Table S1 (Continued).

\begin{tabular}{|c|c|c|c|c|}
\hline Sample & $\mathrm{NB} / \mathrm{g} \mathrm{L}^{-1}$ & Clay / g L ${ }^{-1}$ & $\begin{array}{l}\text { Basal spacing } \\
/ \mathrm{nm}\end{array}$ & $\begin{array}{l}\text { Volume occupancy of the NB } \\
\text { lamellar phase in the colloid }\end{array}$ \\
\hline \multirow[t]{8}{*}{ NB-FH } & \multirow[t]{3}{*}{30} & 20 & 49 & 0.52 \\
\hline & & 30 & 40 & 0.42 \\
\hline & & 40 & 36 & 0.38 \\
\hline & \multirow[t]{5}{*}{40} & 5 & 71 & 1.0 \\
\hline & & 10 & 64 & 0.90 \\
\hline & & 20 & 51 & 0.72 \\
\hline & & 30 & 43 & 0.61 \\
\hline & & 40 & 35 & 0.49 \\
\hline \multirow[t]{20}{*}{ NB-TSM } & \multirow[t]{5}{*}{10} & 5 & 102 & 0.36 \\
\hline & & 10 & 80 & 0.32 \\
\hline & & 20 & 62 & 0.22 \\
\hline & & 30 & 47 & 0.17 \\
\hline & & 40 & 45 & 0.16 \\
\hline & \multirow[t]{5}{*}{20} & 5 & 89 & 0.63 \\
\hline & & 10 & 71 & 0.39 \\
\hline & & 20 & 55 & 0.32 \\
\hline & & 30 & 47 & 0.39 \\
\hline & & 40 & 45 & 0.40 \\
\hline & \multirow[t]{5}{*}{30} & 5 & 70 & 0.74 \\
\hline & & 10 & 60 & 0.70 \\
\hline & & 20 & 45 & 0.48 \\
\hline & & 30 & 48 & 0.51 \\
\hline & & 40 & 43 & 0.46 \\
\hline & \multirow[t]{5}{*}{40} & 5 & 70 & 0.99 \\
\hline & & 10 & 67 & 0.95 \\
\hline & & 20 & 57 & 0.80 \\
\hline & & 30 & 46 & 0.65 \\
\hline & & 40 & 39 & 0.55 \\
\hline
\end{tabular}


Table S2. Value of exponent $\beta$ in the power low of $d \propto c_{\mathrm{NB}}{ }^{\beta}$.

\begin{tabular}{lll}
\hline Clay / $\mathrm{g} \mathrm{L}^{-1}$ & Sample & $\beta$ \\
\hline 5 & NB-Hect & -0.35 \\
& NB-Sapo & -0.22 \\
& NB-FH & -0.30 \\
& NB-TSM & -0.30 \\
10 & NB-Hect & -0.01 \\
& NB-Sapo & -0.15 \\
& NB-FH & -0.16 \\
& NB-TSM & -0.15 \\
& NB-Hect & -0.07 \\
& NB-Sapo & -0.09 \\
& NB-FH & -0.09 \\
30 & NB-TSM & -0.11 \\
& NB-Hect & -0.05 \\
& NB-Sapo & -0.18 \\
& NB-FH & -0.06 \\
40 & NB-TSM & -0.05 \\
& NB-Hect & -0.02 \\
& NB-Sapo & 0.00 \\
& NB-FH & -0.07 \\
& NB-TSM & -0.04 \\
\hline
\end{tabular}


(I) Niobate - Hectorite
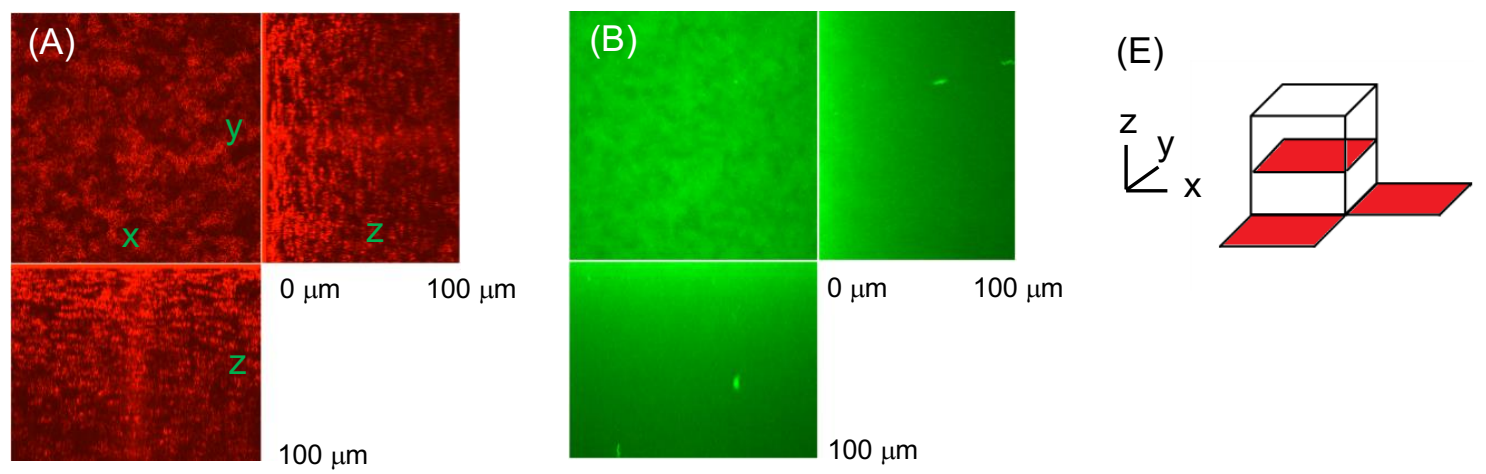

(II) Niobate - Fluorohectorite
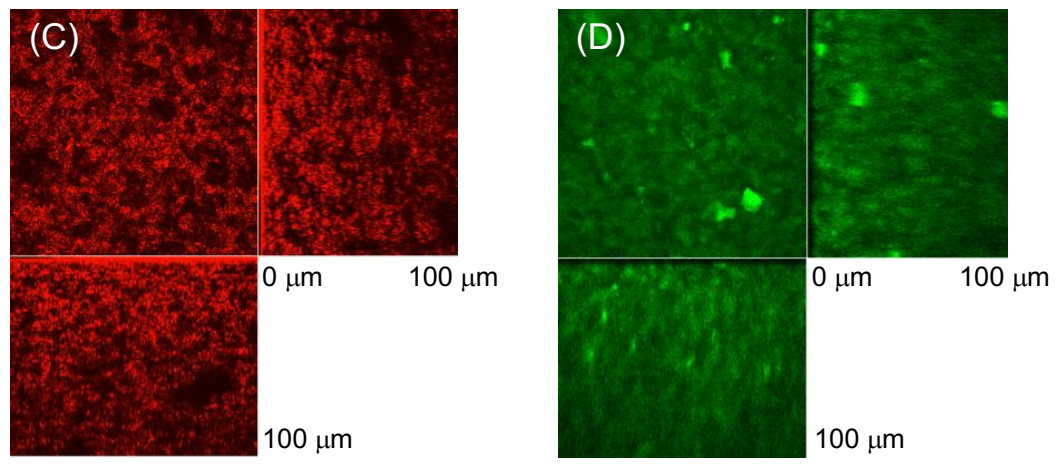

Figure S3. Cross-sectional images obtained by CLMS for (I) NB-Hect $\left(10 \mathrm{~g} \mathrm{~L}^{-1}-5 \mathrm{~g}\right.$ $\left.\mathrm{L}^{-1}\right)(\mathrm{A}, \mathrm{B})$ and (II) NB-FH $\left(10 \mathrm{~g} \mathrm{~L}^{-1}-5 \mathrm{~g} \mathrm{~L}^{-1}\right)$ binary colloids $(\mathrm{C}, \mathrm{D})$. CLMS images obtained by scattering mode $(A, C)$ and fluorescent mode $(B, D)$ are shown in red and green, respectively. Cross-sectional images are extracted from $130 \mu \mathrm{m}(\mathrm{x}$-length $) \times 130$ $\mu \mathrm{m}$ (y-length) $\times 100 \mu \mathrm{m}$ (z-depth) volume for each mode. Three types of cross-sectional images $(x-y, y-z, x-z)$ are shown for each data. Cross-sections selected are indicated by schematic model (E). The $\mathrm{x}-\mathrm{y}$ cross sectional images are at $50 \mathrm{~mm} z$-depth. 
NB-Hect

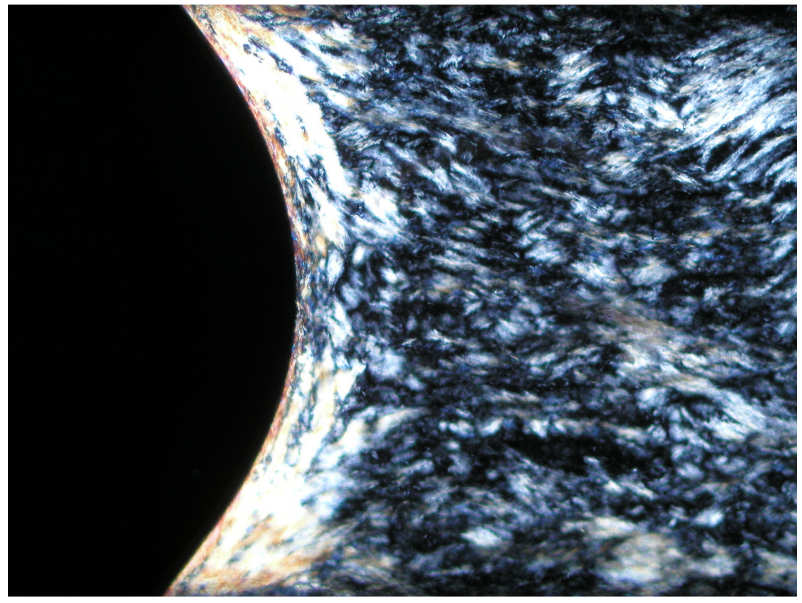

$\mathrm{NB}-\mathrm{FH}$

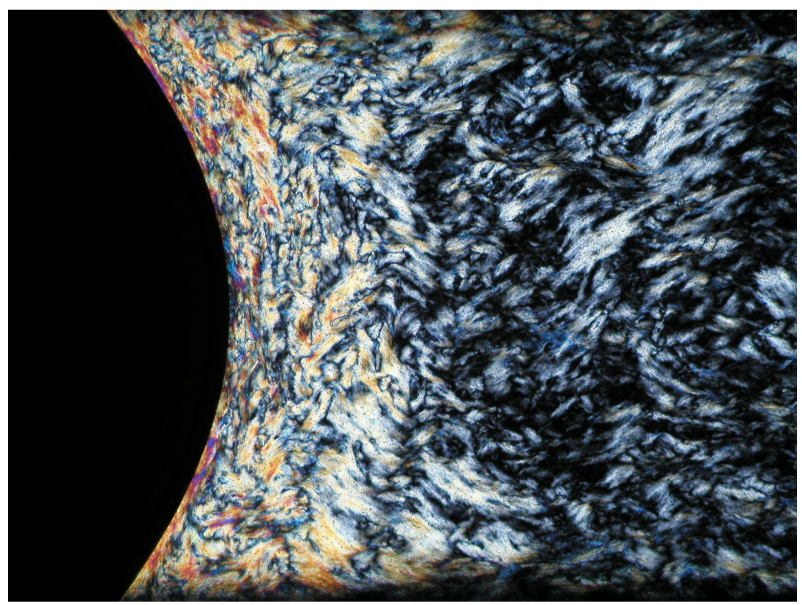

$500 \mu \mathrm{m}$

Figure S4. Polarized optical microscope (POM) images of NB-Hect and NB-FH binary colloids recorded on an Olympus BX-51 optical microscope. The niobate and clay nanosheet concentrations are both $20 \mathrm{~g} \mathrm{~L}^{-1}$ for both of the samples. The samples were confined in $0.2-\mathrm{mm}$ thick flat capillaries. 